\title{
Case selection in macular relocation surgery for age related macular degeneration
}

\author{
D Wong, P Stanga, M Briggs, P Lenfestey, E Lancaster, K K Li, K S Lim, C Groenewald
}

Br J Ophthalmol 2004;88:186-190. doi: 10.1136/bjo.2003.019273

Background: To date there has been no randomised controlled trial demonstrating the safety and efficacy of macular relocation surgery (MRS) for age related macular degeneration (AMD). Vision can be improved in some patients and made worse in others despite successful surgery or because of complications.

Purpose: To determine which patients would benefit from MRS.

Methods: Twenty nine patients with exudative AMD took part in a prospective, non-comparative, interventional study. Macular relocation surgery involved phacoemulsification, vitrectomy, $360^{\circ}$ retinotomy, excision of choroidal neovascular membrane, and macular relocation using an infusion of 5-fluorouracil and low molecular weight heparin as adjuvant to prevent proliferative vitreoretinopathy. Patients underwent protocol refraction preoperatively and six-monthly postoperatively by designated optometrists. Preoperative fundus fluorescein angiograms were read by masked observers and the lesions were classified according to a set protocol. The main outcome measures were visual improvement, final vision of better than $20 / 400$, reading speed, critical print size. Logistic and multiple stepwise linear regressions were used to identify independent factors which predicted the main outcomes. Results: Preoperative visual acuity (20/120 or worse) and lesion type (predominantly classic or submacular haemorrhage) were significantly associated with visual improvement (coefficient of regression $B=26.8, p<0.001$ and $B=14.9$ with $p=0.045$ respectively). There were no significant independent factors which predicted a final distance logMAR visual acuity of $1.3(20 / 400)$ or any arbitrary definition of blindness.

Conclusions: The study showed that it was possible to select cases that were more likely to experience an improvement in vision following MRS.

A lthough many studies have shown that macular relocation surgery (MRS) improved vision in a number of patients, they have also shown that vision can be made worse in some cases. The visual outcome of surgery has been compared with natural history data. The Committee on Procedures Assessment Retina Panel concluded that "to date [March 2000] no strong evidence exists to answer the questions about the effectiveness of macular relocation."1 When we started MRS in 2000, we considered it a priority to identify those patients who were likely to experience improvement in vision. This information could form the basis of power calculations for a prospective randomised controlled trial (PRCT).

The aim of this study was therefore to identify independent variables which might predict the visual outcome in MRS for age related macular degeneration (AMD).

\section{PATIENTS AND METHODS \\ Patients}

From December 2000 to May 2002, twenty nine consecutive patients with AMD underwent MRS using 5-fluorouracil (5FU) and low molecular weight heparin (LMWH) as adjunctive treatment in a prospective, non-comparative interventional pilot study. This study was approved by the local ethics committee. Selection criteria included patients age 60 years or over, signs of age related macular changes, including soft drusens and retinal pigment epithelial changes, and subfoveal choroidal neovascularisation ( $\mathrm{CNV}$ ) in the eye to be treated. All patients were given information on the options of treatment (including photodynamic therapy and no treatment) and a full explanation of the developing nature of the surgery. All patients were given at least one week to decide whether to undergo MRS. The logMAR visual acuity was measured following refraction by designated optometrists using a standardised protocol ${ }^{2}$ All patients had preoperative fundus colour photography and fluorescein angiography, orthoptic assessment, and A-mode ultrasound biometry. Before December 2000, we carried out limited relocation surgery. ${ }^{3}$ The 29 consecutive cases represented all the MRS for AMD performed in our unit up to May 2002.

\section{Surgery}

1. Macular relocation surgery

The surgery began with phacoemulsification and intraocular lens implantation (Acrysof MA30BM, Alcon, Fort Worth, TX, USA) using balanced salt solution. Then a 3 port pars plana vitrectomy was carried out using an infusion of Hartmann solution with added 5-FU $(200 \mu \mathrm{g} / \mathrm{ml})$ and LMWH $(5 \mathrm{IU} / \mathrm{ml})$. The posterior hyaloid was detached and the vitreous base was trimmed closely with a bimanual technique of transillumination and deep indentation using the light pipe (fig 1). The retina was detached using a 41 gauge needle (DORC 1270 0.1, Zuidland, the Netherlands) and the infusion of balanced salt solution via a viscous fluid injector (Accurus 8065-740254, Alcon). A $360^{\circ}$ retinotomy was made using the high speed cutter and transillumination and deep indentation described above. Once the retinotomy was completed, the temporal retina was reflected and the CNV membrane was removed using intraocular forceps. Then the retina was rotated using a single-handed technique by means of the vitreoretinal brush (BD Visitec 585139, Sarasota, USA) through which Perfluorodecalin was injected once the fovea was in the desired location. We moved the fovea superonasally and at least one disc diameter from the edge of the bed of the excised CNV. Endophotocoagulation to the $360^{\circ}$ retinotomy was carried out using an illuminated laser pipe

Abbreviations: $A M D$, age related macular degeneration; $C N V$, choroidal neovascularisation; FFA, fundus fluorescein angiography; $\mathrm{LMWH}$, low molecular weight heparin; MRS, macular relocation surgery; PRCT, prospective randomised controlled trial 


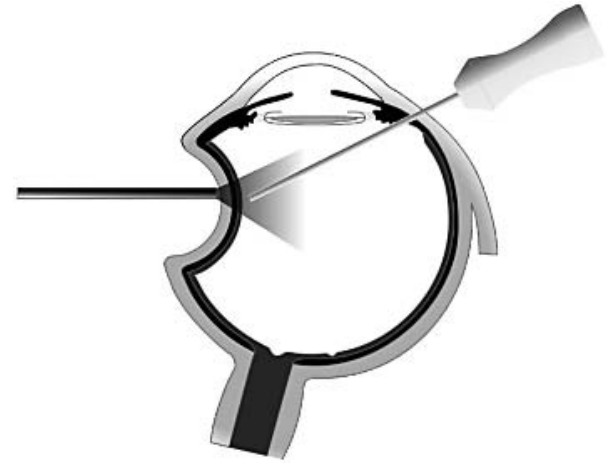

Figure 1 The light pipe is used as a scleral indentor. The transilluminated light provides good illumination for trimming the vitreous base and carrying out the $360^{\circ}$ retinotomy, and enables the surgeon to carry out these procedures bimanually with better control.

(EndoOcular Probe, HGM Inc., Salt Lake City, USA) and a scleral indentor. A direct perfluorodecalin, silicone oil ( 1000 mPas) exchange was carried out using the viscous fluid injection pump and the passive aspiration of a flute needle.

\section{Counter-rotation muscle surgery and removal of oil}

Postoperatively, the patients were instructed to posture at home lying in bed, alternating left or right side down for 14 days. Ten to twelve weeks postoperatively, counterrotation muscle surgery combined with silicone oil removal was performed. The oblique and rectus muscle surgery was done according to the description of Neugebauer and Fricke. ${ }^{4}$ Silicone oil was removed via a 3 ports pars plana approach and an internal search was made for any retinal breaks. All surgeries in this series were carried out by a single surgeon (DW).

\section{Visual function assessment}

All patients had protocol refraction and $\log$ MAR distance visual acuity measurement preoperatively and at 6, 12, and 18 months postoperatively. The Early Treatment Diabetic Retinopathy Study (ETDRS) chart (Lighthouse Television Products, NY, USA) was used. Best corrected visual acuity was measured at $2 \mathrm{~m}$. The score was the total number of letters read correctly plus 15. If the patients saw fewer than 20 letters, they were tested with the top three lines at $1 \mathrm{~m}$. The score then was the total number of letters read at $2 \mathrm{~m}$ plus the number of letters read at $1 \mathrm{~m}$.

Between June and December of 2002, all patients attending for their protocol visits were also tested for their near vision. The MNread charts were used with a +2.50 dioptre addition and $40 \mathrm{~cm}$ reading distance. The logMAR near visual acuity, reading speed, and critical print size were recorded. The Goldmann visual fields were scanned into a computer, the area encompassed by the 4-IV-e was recorded. Each measurement was calibrated against a standard length line drawn on the Goldmann visual field plot. The area was given in square units which were not an absolute but a relative measurement of the size of the visual fields. Contrast sensitivity, using Pelli-Robson charts and colour vision, using the City University Plates, were also evaluated.

\section{Fluorescein angiography}

The preoperative fundus fluorescein angiography (FFA) were masked and numbered. These were graded by two investigators (MB and PL) masked to the clinical outcome of the patients. The FFA were independently read and the lesion types classified according to a set of criteria. The following lesion components were recognised: classic CNV, occult CNV, elevated blocked hyperfluorescence, blood and pigment epithelial detachment. Each observer recorded the presence or absence of each component and judged whether it occupied more than $50 \%$ of the total area of the lesion. Based on the lesion components, the angiograms were classified as predominantly classic (where the classic CNV constituted more than $50 \%$ of the lesion), haemorrhagic (where virtually the whole lesion consisted of retinal blood) and minimally classic (where the classic CNV constituted less than $50 \%$ of the lesion). The greatest linear dimension was measured using a graticule on the FFA and calculated using the known magnification factor of the fundus camera.

\section{RESULTS}

The mean (standard deviation) age of the patients was 75 (SD 6) years (range 63-90). The mean duration between onset of symptoms and MRS was 6.1 (SD 4.4) months (range 2-21). Fifteen patients had predominantly classic lesion, eight had minimally classic, and six patients had submacular haemorrhages.

\section{Follow up}

All patients completed a minimum of six months follow up. The mean follow up was 12.3 (SD 4.5) months (range 6.722.8). One patient failed to attend the 12 month follow up but attended the 18 month appointment. One patient attended for his 12 month examination but because of time pressure, the contrast sensitivity and the colour vision were not measured. One patient missed the 18 month appointment.

\section{Visual function}

The distance visual acuities are summarised in table 1 and figure 2. There was no significant difference between the preoperative and the postoperative visual acuities at six months ( $\mathrm{p}=0.50$ by paired $t$ test). There was no correlation between preoperative and postoperative visual acuities $(r=0.19$ and $\mathrm{p}=0.32)$. A Kaplan-Meier plot is shown in figure 3 with survival being arbitrarily defined as a lost 15 or more ETDRS letters (comparing last to preoperative logMAR visual acuity). The median survival was 22.8 months.

The visual function at the last follow up is summarised in table 2 . The reading acuity and the critical print size were correlated to the distance visual acuities $(r=0.79, \mathrm{p}<0.001$ and $r=0.70, \mathrm{p}<0.001$ respectively). The reading acuity however, was slightly better than the distance visual acuity and the difference was significant (paired $t$ test, $\mathrm{p}=0.002$ ). The area of the 4-IV-e isoptre in the operated eye was significantly smaller than that of the fellow eye (paired $t$ test comparing means $\mathrm{p}<0.001$ ).

\section{Retinal detachment and proliferative vitreoretinopathy}

With the exception of the phacoemulsification part of the surgery, the cocktail ${ }^{5}$ of 5-FU and LMWH in Hartmann

\begin{tabular}{|c|c|c|c|}
\hline & Preoperative & 6 months & Last follow up \\
\hline $\begin{array}{l}\text { Mean (SD) distance } \\
\text { visual acuity } \\
\text { (range) }\end{array}$ & $\begin{array}{l}0.98(0.42) \\
(0.12-1.7)\end{array}$ & $\begin{array}{l}0.93(0.26) \\
(0.281 .4)\end{array}$ & $\begin{array}{l}0.97(0.29) \\
(0.28-1.4)\end{array}$ \\
\hline $\begin{array}{l}\% \text { increase } \geqslant 15 \\
\text { ETDRS letters }\end{array}$ & - & $28 \%$ & $31 \%$ \\
\hline $\begin{array}{l}\text { Within } \pm 15 \text { ETDRS } \\
\text { letters }\end{array}$ & - & $48 \%$ & $41 \%$ \\
\hline $\begin{array}{l}\% \text { decrease } \geqslant 15 \\
\text { ETDRS lefters }\end{array}$ & - & $24 \%$ & $28 \%$ \\
\hline
\end{tabular}


A
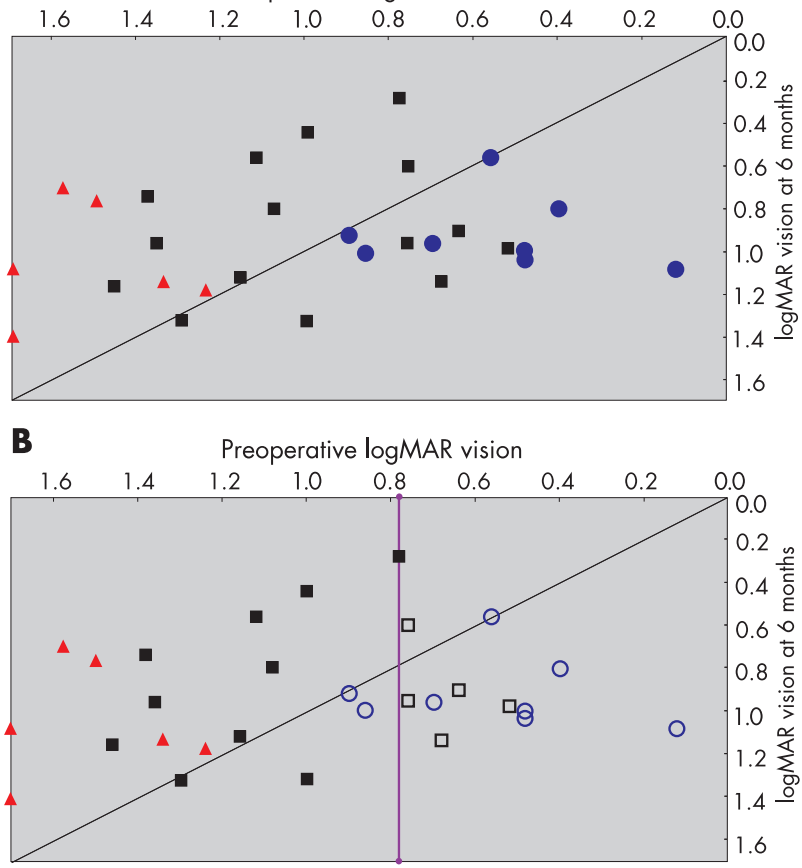

Figure 2 (A) A plot of preoperative against six months logMAR visual acuity. Red triangles, haemorrhagic lesions; black squares,

predominantly classic lesions; blue circles, minimally classic lesions. The diagonal line is the line of no change. Patients with improvement are shown above and to the left of this line. (B) The same plot showing selection of patients. The vertical line is the preoperative visual cut off of 0.78 . Patients to the left of this line are selected. Solid symbols, patients selected; empty symbols, patients not selected. By selecting all predominantly classic and haemorrhagic cases with a preoperative vision of 0.78 or worse, all patients with visual improvement are included and all but two patients with visual deterioration are excluded.

solution was used as the infusion fluid throughout the relocation surgery. The mean duration of the operation was two hours 33 minutes. Of the 29 patients, there had been three cases of RRD 2 of which were associated with PVR. The rate of retinal detachment was $10.3 \%$ (95\% CI 2 to 27 ) and PVR was $6.9 \%$ (95\% CI 1 to 23 ).

\section{Fundus fluorescein angiography}

There were three disagreements in the classification of the lesions. The concordance was analysed using cross tabulation and the kappa $=0.735(\mathrm{p}<0.001)$. Following discussion and while the readers were still masked to the visual outcomes, a

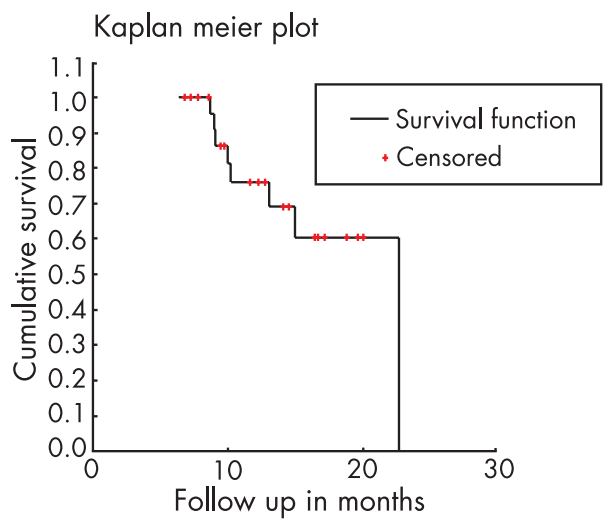

Figure 3 Survival defined as not having lost 15 or more letters at the final follow up visit.
Table 2 Visual function at the last follow up

\begin{tabular}{lll}
\hline & Mean (SD) & Range \\
\hline Duration of follow up in months & $12.3(4.5)$ & $6.7-22.8$ \\
Distance logMAR visual acuity & $0.97(0.29)$ & $0.28-1.4$ \\
Near logMAR visual acuity & $0.85(0.30)$ & $0.32-1.4$ \\
Reading speed in words per minute & $62(43)$ & $0-177$ \\
Critical print size in logMAR units & $1.03(0.24)$ & $0.4-1.4$ \\
$\begin{array}{l}\text { Area of Goldmann 4-IV-e of the operated } \\
\text { eye in square units }\end{array}$ & $113(56)$ & $12-223$ \\
$\begin{array}{l}\text { Area of Goldmann 4-IV-e of the fellow } \\
\text { eye in square units }\end{array}$ & $203(64)$ & $24-301$ \\
$\begin{array}{l}\text { Pelli-Robson: contrast sensitivity score } \\
\text { City University test: No of colour plates }\end{array}$ & $16.5(8.3)$ & $0-30$ \\
\hline
\end{tabular}

The Goldmann visual fields were scanned into a computer and the area of the 4-IV-e was measured in square units using a graphics programme.

consensus was reached for the three discrepancies. The two readers classified the 29 cases into 15 predominantly classic, eight minimally classic, and six haemorrhagic lesions. The correlation between the lesion size (greatest linear dimension) as measured by the two observers was also good; the coefficient of correlation $r=0.69$ and the $\mathrm{p}<0.001$. The mean size as measured on the FFA, expressed as greatest linear dimension (GLD) was 5409 (SD 3407) $\mu \mathrm{m}$ (range 1407$16776 \mu \mathrm{m})$. The largest lesions were observed in patients with submacular haemorrhages, the mean GLD of the three haemorrhagic lesions being $11720 \mu \mathrm{m}$.

\section{Statistical analyses}

\section{Preoperative Vision}

Figure 4 plots the change in visual acuity versus the preoperative visual acuity. The trend line for this plot has a negative gradient suggesting the change in visual acuity might be inversely related to the preoperative visual acuity. The surgery seemed to have an averaging effect. Patients with poor preoperative vision tended to be made better and visa versa. To determine whether this was a genuine effect or whether this was simply a natural tendency of "regression towards the means" ${ }^{67}{ }^{7}$ we tested the correlation between the change in the visual acuity with the average of the pre- and postoperative visual acuities ${ }^{8}$ (Bland Altman Plot). The correlation coefficient $r=0.43$ and was significant with $\mathrm{p}=0.021$.

For case selection, we might choose a level of vision of 0.78 $(20 / 120)$ as a cut off. We find that a preoperative vision of 0.78 or worse was significantly associated with improvement (defined as any increase in the number of ETDRS letters at six months) with $\chi^{2}$ value of $12.9, \mathrm{p}<0.001$. The association

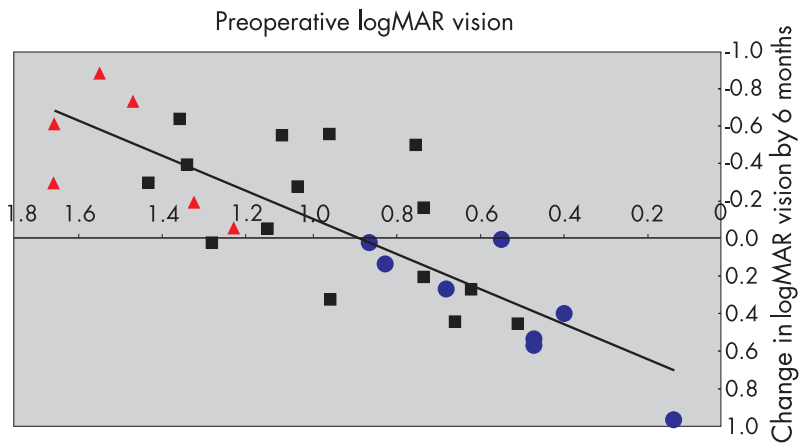

Figure 4 Plotting change in logMAR visual acuity against preoperative $\log M A R$ visual acuity. Note with logMAR vision any improvement is negative. Thus, patients above the $x$ axis had improvement and below had deterioration. The trend line has a negative slope, suggesting that the worse the preoperative vision, the greater the improvement. 
with "significant" improvement (defined as a gain of 15 or more ETDRS letters) gave a $\chi^{2}$ value of 9.3, $\mathrm{p}=0.002$.

\section{Lesion type}

Figure 2A used symbols to identify the lesion type of each individual patient as predominantly classic, haemorrhagic, or minimally classic. This gives a strong impression that the outcome for patients with the three different lesion types are distinct - that haemorrhagic cases tended to have poor initial vision and to have improved by six months, that the predominantly classic cases have intermediate initial vision that tended to be stabilised, and that minimally classic type cases have good initial vision that tended to have deteriorated at six months. Comparing preoperative vision in the three types by a Kruskal-Wallis test gives $\chi^{2}=16.9$, df $=2$, $\mathrm{p}=0.0003$. Therefore, there is strong evidence that the three clinical types have different preoperative vision. Comparing the change from preoperative to six month vision between the three clinical types by a Kruskal-Wallis test gives $\chi^{2}=12.02, \mathrm{df}=2, \mathrm{p}=0.002$-very strong evidence that the three types behave differently.

If the predominantly classic and haemorrhagic types were grouped together, we found that lesion type was significantly associated with improvement $\left(\chi^{2}\right.$ was 11.8 with $\left.\mathrm{p}=0.001\right)$ and "significant" improvement $\left(\chi^{2}\right.$ was 5.8 with $\left.\mathrm{p}=0.016\right)$.

\section{Case selection}

The data in this study can be used to plan for a prospective randomised controlled trial. Multiple stepwise linear regression analyses showed that the two variables (preoperative vision and lesion type) were independent; each had a separate and significant effect on change (the number of ETDRS letters gained or lost at six months). The coefficient of regression $\mathrm{B}$, for predicting change was $\mathrm{B}=26.8$ with $\mathrm{p}<0.001$ for preoperative vision and $\mathrm{B}=14.9$ with $\mathrm{p}=0.045$ for lesion type.

Figure 2B illustrates that by selecting patients with preoperative vision of $0.78(20 / 120)$ or worse and by selecting patients with predominantly classic and haemorrhagic lesions, all the patients with visual improvements would have been included and, conversely, all but two patients with visual deterioration would have been excluded.

When lesion size, duration of symptoms, age of patient, duration of surgery, and chronological order of surgery (as a reflection of learning curve) were tested, none was found to predict improvement. There were no independent factors which predicted final visual acuity (at 20/400 or any other level), reading speed, and reading acuity.

\section{DISCUSSION}

The feasibility of relocating the macula was first demonstrated by Machemer and Steinhorst in 1992. ${ }^{\circ}$ In the last three years, there have been over 50 publications in peer reviewed journals on macular relocation surgery (involving different techniques). It has been asserted that MRS is the only treatment in AMD that aims to restore lost vision ${ }^{10-13}$ but to date, there have not been any series which showed that the postoperative vision was significantly better than the preoperative vision. Lai et $a^{14}$ found in a series of 15 consecutive patients that the reading vision was significantly better at six months when compared with preoperatively (mean logMAR vision improved from 0.54 to $0.40, \mathrm{p}=0.02$ ). By 12 months however, the mean near vision was back to $0.54 .{ }^{14}$ The results of a consecutive series of 90 cases reported from Cologne showed that the distance visual acuity increased by 15 or more letters in $27 \%$, remained stable in $41 \%$, and deteriorated by 15 or more letters in $32 \%$ at 12 months follow up. ${ }^{15}$ Although it might be correct to say that MRS aims to improve vision, ${ }^{13}$ in practice there is no evidence to suggest it does so consistently. Stated simply, the improvement in some patients is offset by deterioration in others. The best results were perhaps those reported by Pertile $e t$ al $^{16}$ in a consecutive series of 50 cases. They concluded in their report that "further investigations are necessary to determine which patients will have the most benefit from this complex therapeutic method". Indeed we believe that this is the most important research question. Case selection is the key to power calculations for a prospective randomised controlled trial which has yet to be performed.

In this study we have reported on all cases of AMD treated with MRS in this unit over an 18 month period. The patients were highly motivated, and even though most were elderly and some travelled a long way, the follow up was good. The use of the British cocktail of 5-FU and heparin, ${ }^{5}$ protocol refraction, designated optometrists, $\log$ MAR distance and near charts at planned intervals, along with masked readers of fluorescein angiograms, are important features of our study.

In terms of the proportion of patients improving, remaining stable, and deteriorating (using a change of 15 ETDRS letters at $1 \mathrm{~m}$ or a doubling of visual angle), the results at six months and at last follow up were largely comparable with those reported in other series, even though the preoperative vision in our patients tended to be poorer and the duration of symptoms tended to be longer. ${ }^{14-17}$ The preponderance of predominantly classic lesions in our series was because many patients were initially referred for photodynamic treatment.

Pieramici, in a series of limited translocation cases, found that the preoperative visual acuity was significantly correlated with postoperative visual acuity. ${ }^{17}$ Although there was a large number of patients lost to follow up, the data seemed to infer that to obtain good postoperative visual results, patients with good preoperative vision should be selected. In our series, no correlation was found between the preoperative and the postoperative visual acuity.

Instead, we found a relationship between preoperative visual acuity and the change in visual acuity by six months. This relationship is inversed-that is, the worse the preoperative vision the greater the improvement, and this occurred more than was predicted by chance.

The lesion characteristic was also an independent variable and had a separate influence on the change in vision. Patients with minimally classic lesion tendeded to have better visual acuities preoperatively and poorer visual acuities at six months and at last follow up. They also tended to have a more insidious disease. ${ }^{18}$ The overlying photoreceptors might already be damaged by the chronic nature of the disease, and with the further insult of surgery, vision was made worse. The efficacy of surgery for minimally classic lesions is being studied in an international multicentre prospective randomised controlled trial organised by the Cologne group (Macular Relocation in Age related Neovascular disease (MARAN) study). Outside the context of a PRCT however, it might be difficult for us to justify surgery on minimally classic lesions based on our results. We are hesitant to extrapolate from our results as most of the patients have a long history of visual symptoms. Other centres may have a different experience with minimally classic lesions: by treating early, patients might benefit from stabilisation even if surgery did not produce improvement.

The finding of the field defect is not new. ${ }^{19-22}$ It might be partly explained by the fact that the optic nerve head is not at the geometric centre of the retina or by extensive peripheral laser retinopexy. ${ }^{23}$ Whether the rotation of the retina through large angles might damage the nerve fibre layer, we can only speculate. We now routinely perform Goldmann perimetry preoperatively as a baseline measurement. It might be argued that patients with substantial field loss in the fellow eye should not be selected for MRS. We suggest that all patients, 
especially one eyed patients, should be counselled on the possibility of loss of peripheral vision as a result of surgery. ${ }^{24}$

One of the most serious complications of MRS is retinal detachment and proliferative vitreoretinopathy (PVR). ${ }^{11}$ In the past, we have shown the value of the adjuvant 5-FU and heparin in preventing PVR in high risk patients ${ }^{25}$ We are encouraged by the relatively low rate of retinal detachment and PVR in this series, taking into account the fact that these were our first cases.

As our study was a non-comparative one, the results cannot be used to justify treatment, but they can help the planning for prospective randomised controlled trials. Surgery appeared to have an averaging effect. Patients with good preoperative visual acuity tended to be made worse and patients with poor preoperative visual acuity tended to be improved. We have identified two independent factors, namely preoperative visual acuity and lesion type. The two factors appear to predict improvement. By selecting patients with poorer vision, not amenable to any other proved forms of treatment, ${ }^{26}$ it would be possible to use natural history as a control group in a PRCT. The numbers involved in such a trial would be relatively small and feasibly recruited from one or two centres. The authors are convinced that macular relocation surgery has a role to play in selected patients with age related macular degeneration. It is very much hoped that such a randomised trial will be conducted to provide the evidence base for MRS.

\section{ACKNOWLEDGEMENTS}

The authors would like to acknowledge Mr Ian Campbell for the helpful statistical advice, and Mr Simon Harding and Mr Ian Pearce for their continued support in recruiting patients. This study was supported by the Ridley Foundation and by the Research and Development Fund of the Royal Liverpool University Hospital.

\section{Authors' affiliations}

D Wong, P Stanga, M Briggs, P Lenfestey, E Lancaster, K K Li, K S Lim, C Groenewald, St Paul's Eye Unit, Royal Liverpool University Hospital, Liverpool, UK

P Stanga, Experimental Ophthalmology Unit, Department of Medicine, University of Liverpool, UK

All correlation quoted in this paper are Pearson's and all $p$ values are two tailed.

Correspondence to: Mr David Wong, Consultant Ophthalmologist, St Paul's Eye Unit, Royal Liverpool University Hospital, Prescot Street, Liverpool L7 8XP, UK; shdwong@liverpool.ac.uk

Accepted for publication 13 May 2003

\section{REFERENCES}

1 Macular translocation. American Academy of Ophthalmology. Ophthalmology 2000;107:1015-8.
2 Photodynamic therapy of subfoveal choroidal neovascularization in agerelated macular degeneration with verteporfin: one-year results of 2 randomized clinical trials-TAP report. Treatment of age-related macular degeneration with photodynamic therapy (TAP) Study Group. Arch Ophthalmol 1999:117:1329-45.

3 Wong $\mathrm{D}$, Lois $\mathrm{N}$. Foveal relocation by redistribution of the neurosensory retina. Br J Ophthalmol 2000;84:352-7.

4 Neugebauer A, Fricke J, Aisenbrey S, et al. Cyclotropia in macula translocation. Possible eye muscle surgery solutions. Ophthalmologe 2002;99:10-4

5 Sebag J. Shaken not stirred. Ophthalmology 2001;108:1177-8.

6 Bland JM, Altman DG. Regression towards the mean. BMJ 1994;308:1499.

7 Bland JM, Altman DG. Some examples of regression towards the mean. BMJ 1994;309:780.

8 Bland JM, Altman DG. Statistical methods for assessing agreement between two methods of clinical measurement. Lancet 1986;1:307-10.

9 Machemer R, Steinhorst UH. Retinal separation, retinotomy, and macular relocation: II. A surgical approach for age-related macular degeneration? Graefe Archive For Clinical and Experimental Ophthalmology 1993;231:635-41.

10 Machemer R. Macular translocation. Am J Ophthalmol 1998; 125:698-700.

11 Eckardt C, Eckardt U, Conrad HG. Macular rotation with and without counterrotation of the globe in patients with age-related macular degeneration. Graefes Arch Clin Exp Ophthalmol 1999;237:313-25.

12 Lewis H, Kaiser PK, Lewis S, et al. Macular translocation for subfoveal choroidal neovascularization in age-related macular degeneration: a prospective study. Am J Ophthalmol 1999;128:135-46.

13 Cekic O, Ohii M, Hayashi A, et al. Foveal translocation surgery in age-related macular degeneration. Lancet 1999;354:340.

14 Lai JC, Lapolice DJ, Stinnett SS, et al. Visual outcomes following macular translocation with 360-degree peripheral retinectomy. Arch Ophthalmol 2002; 120:1317-24.

15 Aisenbrey S, Lafaut BA, Szurman P, et al. Macular translocation with 360 degrees retinotomy for exudative age-related macular degeneration. Arch Ophthalmol 2002;120:451-9.

16 Pertile G, Claes C. Macular translocation with 360 degree retinotomy for management of age-related macular degeneration with subfoveal choroida neovascularization. Am J Ophthalmol 2002;134:560.

17 Pieramici DJ, De Juan E, Fujii GY, et al. Limited inferior macular translocation for the treatment of subfoveal choroidal neovascularization secondary to age-related macular degeneration. Am J Ophthalmol 2000;130:419-28.

18 Stevens TS, Bressler NM, Maguire MG et al. Occult choroidal neovascularization in age-related macular degeneration. A natural history study. Arch Ophthalmol 1997;1 15:345-50

19 Kubota A, Ohii M, Kusaka S, et al. Evaluation of the peripheral visual field after foveal translocation. Am J Ophthalmol 2001;132:581-4.

20 Terasaki $\mathrm{H}$. Rescue of retinal function by macular translocation surgery in agerelated macular degeneration and other diseases with subfoveal choroidal neovascularization. Nagoya J Med Sci $2001 ; 64: 1-9$

21 Ohii M, Fujikado T, Saito Y, et al. Foveal translocation: a comparison of two techniques. Semin Ophthalmol 1998;13:52-62.

22 Terasaki $H$, Miyake $Y$, Suzuki T, et al. Change in full-field ERGs after macular translocation surgery with 360 degrees retinotomy. Invest Ophthalmol Vis Sci 2002;43:452-7.

23 Frank RN. Visual fields and electroretinography following extensive photocoagulation. Arch Ophthalmol 1975;93:591-8.

24 Gelisken F, Bartz_Schmidt KU. [Visual prognosis and patient selection] Ophthalmologe 2002;99:144-9.

25 Asaria RH, Kon $\mathrm{CH}$, Bunce $\mathrm{C}$, et al. Adjuvant 5-fluorouracil and heparin prevents proliferative vitreoretinopathy: Results from a randomized, doubleblind, controlled clinical trial. Ophthalmology 2001;108:1179-83.

26 National Institute for Clinical Excellence. Final Appraisal Determination Photodynamic therapy for age-related macular degeneration, May 2002. http://www.nice.org.uk/article.asp?a=32774. Accessed 12 June, 2002 